and activates translesion synthesis DNA repair pathway. ${ }^{19}$ Furthermore, cell cycle dependent kinase-9 (CDK9) regulates UBE2A activity by phosphorylating at serine $120 .{ }^{20}$

$U B E 2 A$ regulates the $u$ biquitination of histone $\mathrm{H} 2 \mathrm{~B}$ and proliferating cell nuclear antigen (PCNA) through the cognate E3 ubiquitin ligase RNF20/40 and RAD18, respectively. In addition to its role in transcriptional elongation, histone H2B K120 monoubiquitination plays a crucial role in DNA double strand break (DSB) repairs. ${ }^{21}$ Both these processes describe the role of UBE2A in DNA repair and maintenance of genome integrity. The loss-of-function mutations of UBE2A in advanced phase CML patients may be associated with impaired ubiquitination of $\mathrm{H} 2 \mathrm{~B}$ and PCNA, and hence increased genome instability resulting in the acquisition of additional mutations (Figure 1, signaling paths $\mathrm{C}$ and D). The work by Magistroni et al. ${ }^{5}$ focuses on the latter signaling paths as possibly being at the root of the myeloid transformation. While the mechanisms that control the blastic transformation of CML by UBE2A mutations remain unclear, mutation studies like that of Magistroni et al. do generate hypotheses that should be tested in further studies into BCR-ABL leukemia initiation and propagation.

\section{References}

1. Daley GQ, Van Etten RA, Baltimore D. Induction of chronic myelogenous leukemia in mice by the $\mathrm{P} 210 \mathrm{bcr} / \mathrm{abl}$ gene of the Philadelphia chromosome. Science. 1990;247(4944):824-830.

2. Gambacorti-Passerini CB, Gunby RH, Piazza R, Galietta A, Rostagno $\mathrm{R}$, Scapozza L. Molecular mechanisms of resistance to imatinib in Philadelphia-chromosome-positive leukaemias. Lancet Oncol. 2003;4(2):75-85.

3. Perrotti D, Jamieson C, Goldman J, Skorski T. Chronic myeloid leukemia: mechanisms of blastic transformation. J Clin Invest. 2010;120(7):2254-2264.

4. Mullighan CG, Goorha S, Radtke I, et al. Genome-wide analysis of genetic alterations in acute lymphoblastic leukaemia. Nature. 2007;446(7137):758-764

5. Magistroni V, Mauri M, D'Aliberti D, et al. De novo UBE2A mutations are recurrently acquired during chronic myeloid leukemia progression and interfere with myeloid differentiation pathways.
Haematologica. 2019;104(9):1789-1797.

6. Swatek KN, Komander D. Ubiquitin modifications. Cell Res. 2016;26(4):399-422.

7. Deshaies RJ, Joazeiro CA. RING domain E3 ubiquitin ligases. Annu Rev Biochem. 2009;78:399-434.

8. Gallo LH, Ko J, Donoghue DJ. The importance of regulatory ubiquitination in cancer and metastasis. Cell Cycle. 2017;16(7):634-648.

9. Shen JD, Fu SZ, Ju LL, et al. High expression of ubiquitin-conjugating enzyme E2A predicts poor prognosis in hepatocellular carcinoma. Oncol Lett. 2018;15(5):7362-7368.

10. Seghatoleslam A, Monabati A, Bozorg-Ghalati F, et al. Expression of UBE2Q2, a putative member of the ubiquitin-conjugating enzyme family in pediatric acute lymphoblastic leukemia. Arch Iran Med. 2012;15(6):352-355.

11. Luo H, Qin Y, Reu F, et al. Microarray-based analysis and clinical validation identify ubiquitin-conjugating enzyme E2E1 (UBE2E1) as a prognostic factor in acute myeloid leukemia. J Hematol Oncol. 2016;9(1):125.

12. Anand M, Chodda SK, Parikh PM, Nadkarni JS. Abnormal levels of proinflammatory cytokines in serum and monocyte cultures from patients with chronic myeloid leukemia in different stages, and their role in prognosis. Hematol Oncol. 1998;16(4):143-154.

13. Barreyro L, Chlon TM, Starczynowski DT. Chronic immune response dysregulation in MDS pathogenesis. Blood. 2018;132(15): 1553-1560.

14. Mao JH, Sun XY, Liu JX, et al. As4S4 targets RING-type E3 ligase c$\mathrm{CBL}$ to induce degradation of BCR-ABL in chronic myelogenous leukemia. Proc Natl Acad Sci U S A. 2010;107(50):21683-21688.

15. Reavie L, Buckley SM, Loizou E, et al. Regulation of c-Myc ubiquitination controls chronic myelogenous leukemia initiation and progression. Cancer Cell. 2013;23(3):362-375.

16. Bolton-Gillespie E, Schemionek M, Klein HU, et al. Genomic instability may originate from imatinib-refractory chronic myeloid leukemia stem cells. Blood. 2013;121(20):4175-4183.

17. Shekhar MP, Lyakhovich A, Visscher DW, Heng H, Kondrat N. Rad6 overexpression induces multinucleation, centrosome amplification, abnormal mitosis, aneuploidy, and transformation. Cancer Res. 2002;62(7):2115-2124

18. Garg P, Burgers PM. Ubiquitinated proliferating cell nuclear antigen activates translesion DNA polymerases eta and REV1. Proc Natl Acad Sci U S A. 2005;102(51):18361-18366.

19. Hoege C, Pfander B, Moldovan GL, Pyrowolakis G, Jentsch S. RAD6-dependent DNA repair is linked to modification of PCNA by ubiquitin and SUMO. Nature. 2002;419(51):135-141.

20. Shchebet A, Karpiuk O, Kremmer E, Eick D, Johnsen SA Phosphorylation by cyclin-dependent kinase- 9 controls ubiquitinconjugating enzyme-2A function. Cell Cycle. 2012;11(11):21222127.

21. Nakamura K, et al. Regulation of homologous recombination by RNF20-dependent H2B ubiquitination. Mol Cell. 2011;41(5):515528.

\title{
Six-packed antibodies punch better
}

\section{Christoph Rader ${ }^{1}$ and Adrian Wiestner ${ }^{2}$}

${ }^{1}$ Department of Immunology and Microbiology, The Scripps Research Institute, Jupiter, FL and ${ }^{2}$ Hematology Branch, National Heart, Lung, and Blood Institute, National Institutes of Health, Bethesda, MD, USA

E-mail: CHRISTOPH RADER - crader@scripps.edu

ADRIAN WIESTNER - wiestnera@mail.nih.gov

doi:10.3324/haematol.2019.224196

I $\mathrm{n}$ this issue of the Journal, Oostindie et al. investigate CD37-specific monoclonal antibodies (mAb) engineered to undergo hexamerization. ${ }^{1}$ Efficient hexamer formation is induced by a single amino acid substitution, E430G, in the IgG1 constant domain previously described by the same group. ${ }^{2}$ The modification potentiates complement-dependent cytotoxicity (CDC) against chronic lymphocytic leukemia (CLL) cells in vitro. Next, the authors show that combinations of hexamerizationenhanced $\mathrm{mAb}$ against CD20 and CD37 provide syner- gistic activity. Intriguingly, the CD20- and CD37-targeting $\mathrm{mAb}$ formed mixed hexameric complexes on the cell surface with increased anti-tumor activity.

The anti-CD20 mAb rituximab is a critical component of treatment regimens for many B-cell malignancies. ${ }^{3}$ In combination with chemotherapy, rituximab has been shown to increase response rates, response duration, and overall survival. Single-agent rituximab is quite commonly used in follicular lymphoma and as maintenance therapy in several types of B-cell non-Hodgkin lymphoma (B- 
NHL), including CLL. Compared to other B-NHL, CLL cells have a relatively lower expression of CD20, and single-agent rituximab has limited activity in CLL. Few studies have investigated the combination of two $\mathrm{mAb}$. The combination of rituximab with the anti-CD52 targeting $\mathrm{mAb}$ alemtuzumab yielded a higher rate of complete responses in CLL than had historically been seen with rituximab alone. ${ }^{4}$ However, the manufacturer withdrew alemtuzumab for the treatment of CLL.

Like CD20, the tetraspanin CD37 is an integral membrane protein abundantly expressed on B cells but not on plasma cells or hematopoietic stem cells. ${ }^{5} \mathrm{~T}$ cells, natural killer (NK) cells, granulocytes, and monocytes express low levels of CD37. Tetraspanins are central to membrane organization and play important roles in cell migra- tion and adhesion. ${ }^{6}$ CD37 has been found to co-localize with integrin $\alpha 4 \beta 1$ on $B$ cells and to contribute to cell adhesion and the transduction of survival signals. ${ }^{7}$

Several anti-CD37 antibodies are undergoing clinical investigation in B-cell malignancies. ${ }^{5}$ Otlertuzumab (also called TRU-016), a single-chain variable fragment (scFv) against CD37 linked to the IgG1 Fc fragment, induces apoptosis in CLL cells and mediates antibody-dependent cellular cytotoxicity (ADCC) but not CDC. Otlertuzumab has been shown to have single-agent activity in $\mathrm{CLL},{ }^{8}$ and in combination with bendamustine increased the response rate and prolonged progressionfree survival over single-agent bendamustine. ${ }^{9}$ BI 836826, a chimeric mouse-human $\mathrm{mAb}$ with Fc modifications to increase affinity to Fc $\gamma$ RIIIa effectively mediates ADCC

A

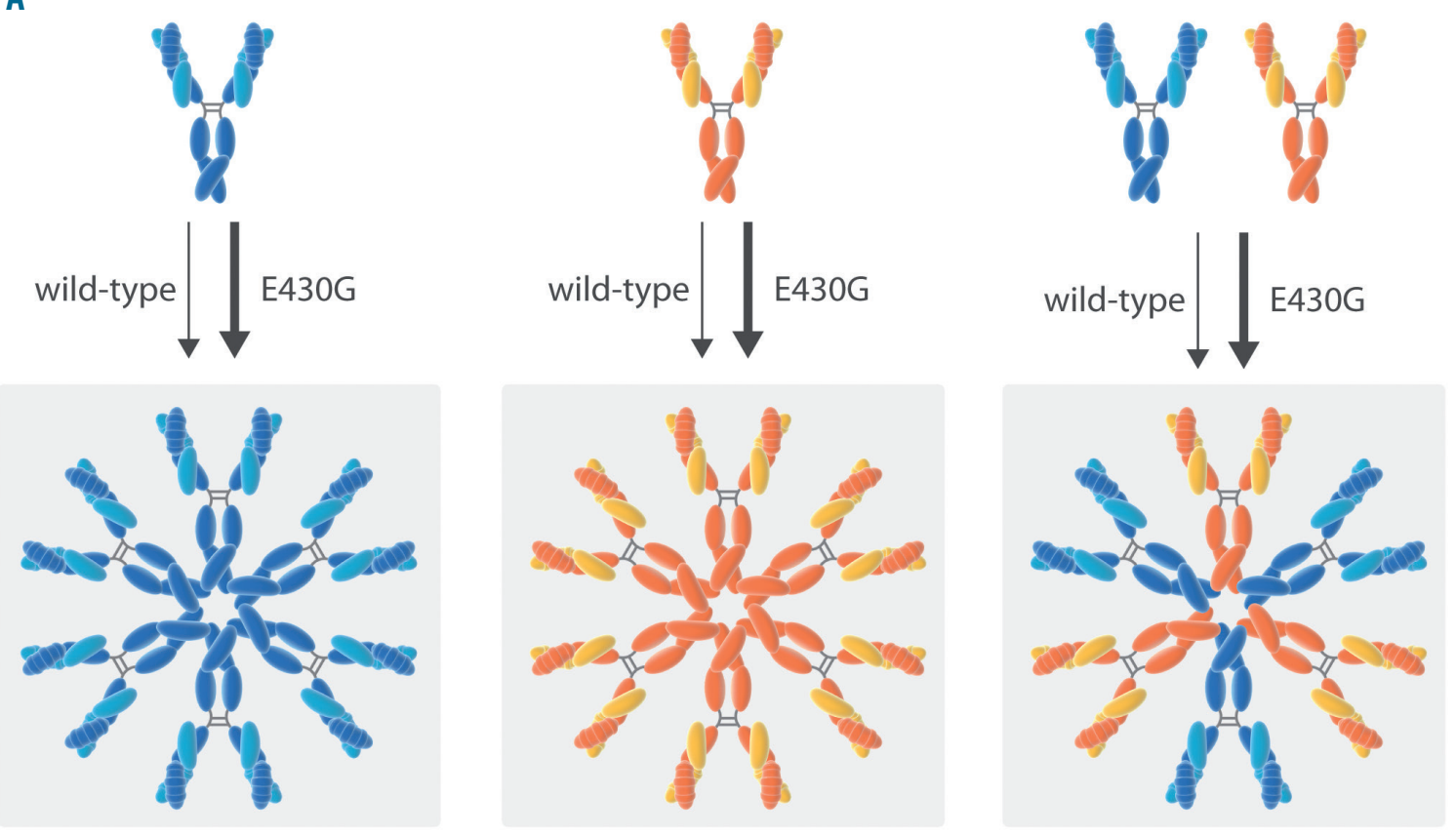

B
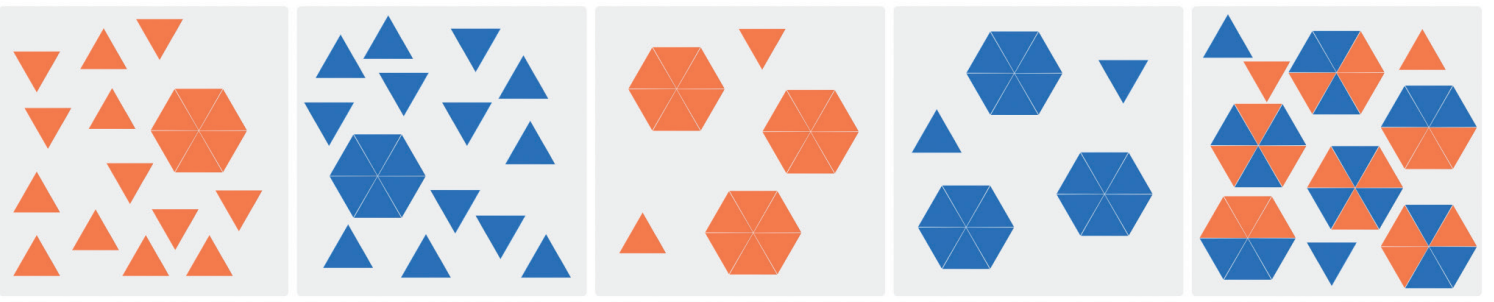

CDC

Figure 1. Hexamerization and hetero-hexamerization of CD20- and CD37-targeting mAb on the cell surface. (A) Shown are CD20-targeting (blue) and CD37-targeting (orange) mAb in IgG1 format. Monomeric in solution, they form hexamers upon cell surface antigen binding. This natural hexamerization of wild-type lgG1 is enhanced by substituting a glutamic acid residue in the IgG1 Fc fragment with a glycine residue (E430G). Mixing CD20- and CD37-targeting mAb leads to the formation of hetero-hexamers in 3:3 (shown here), 4:2, 2:4, 5:1, or 1:5 compositions. (B) Oostindie et al. ${ }^{1}$ show a gradual increase in complement-dependent cytotoxicity (CDC) mediated by wild-type CD37-targeting lgG1 (left) to wild-type CD20-targeting IgG1 (second from left) to CD37-targeting IgG1 with E430G mutation (center) to CD20-targeting IgG1 with E430G mutation (second from right) to mixed CD20-and CD37-targeting IgG1 with E430G mutation (right). This increase in CDC correlates with the density of $\mathrm{C} 1 \mathrm{q}$ docking sites. For simplification, only hetero-hexamers in various 3:3 compositions are shown. 
and also induces apoptosis of CLL cells. In a phase I doseescalation study, BI 836826 was well-tolerated up to doses of $400 \mathrm{mg}$ and had a similar adverse event profile as other Fc-modified antibodies. ${ }^{10}$ The objective response rate was $61.5 \%$ in patients treated at doses $\geq 200 \mathrm{mg}$. Two antibody-drug conjugates and a radioimmunoconjugate targeting CD37 are also undergoing clinical investigation. ${ }^{5}$

Currently, 36 antibody-based cancer therapies approved by the US Food and Drug Administration (FDA), including 4 biosimilars, are on the market. The majority of these treatments are $\mathrm{mAb}$ in IgG1 format that mediate tumor cell killing on their own or in combination with chemotherapy. A key challenge has been the identification of suitable targets for therapeutic $\mathrm{mAb}$ as tumorspecific antigens are rare, and tumor-associated antigens are often expressed on essential healthy cells, lowering the therapeutic index. By contrast, lineage-specific antigens that are expressed on non-essential healthy cells have emerged as preferred targets of therapeutic $\mathrm{mAb}$. A prime example is CD20, which is expressed on healthy and malignant $\mathrm{B}$ cells, and targeted by rituximab (FDA approved in 1997), its biosimilar rituximab-abbs (in 2018), ofatumumab (in 2009), and obinutuzumab (in 2013) for treatment of B-cell malignancies. The same applies to other cell surface antigens, such as CD19, that are restricted to the dispensable B-cell lineage of the hematopoietic system. The mechanism of action (MOA) by which mAb eradicate tumor cells include the induction of apoptosis by interfering with receptor/ligand interactions at the cell surface or by recruiting components of the innate immune system, such as plasma proteins in CDC, NK cells in ADCC, and macrophages in antibody-dependent cellular phagocytosis (ADCP). ${ }^{11}$ All three principle mechanisms of innate immune system recruitment, collectively known as effector functions, involve the Fc fragment of IgG1, a homodimer comprising the hinge and the second $\left(\mathrm{C}_{\mathrm{H}} 2\right)$ and third $\left(\mathrm{C}_{\mathrm{H}} 3\right)$ constant domains of the heavy chain. To mediate CDC, ADCC, and ADCP, the Fc fragment interacts with complement protein $\mathrm{C} 1 \mathrm{q}$ and FcrRIIIa and FcrRIIa receptors, respectively. It also mediates prolonged circulatory half-life through neonatal Fc receptor $(\mathrm{FcRn})$ recycling. All of these mechanisms can be fine tuned by subjecting the Fc fragment to protein or carbohydrate engineering. ${ }^{12}$ In fact, several of the FDAapproved $\mathrm{mAb}$ for cancer therapy have engineered $\mathrm{Fc}$ fragments.

Hexabodies constitute a new class of Fc fragment-engineered therapeutic antibodies. ${ }^{13,14} \mathrm{~A}$ single amino acid substitution in $\mathrm{C}_{\mathrm{H}} 3, \mathrm{E} 430 \mathrm{G}$, enhances the formation of IgG1 hexamers upon cell surface antigen binding (Figure 1A). As such, hexamerization, which was first discovered for membrane-bound wild-type $\operatorname{IgG} 1,{ }^{2}$ facilitates the docking of the hexavalent complement protein $\mathrm{C} 1 \mathrm{q}$ initiating CDC. Indeed, previous studies revealed that CD20targeting IgG1 with the E430G mutation mediate significantly enhanced CDC compared to the parental $\mathrm{mAb} \cdot{ }^{13,14}$ The current study by Oostindie et al. ${ }^{1}$ makes the same case for a CD37-targeting IgG1. In addition, combining hexameric (E430G) CD37-targeting IgG1 with one of the FDA-approved CD20-targeting IgG1 (rituximab, ofatumumab, or obinutuzumab) had a synergistic effect in terms of malignant B-cell lysis by CDC in vitro.
Intriguingly, the authors provide evidence that mixing CD20- and CD37-targeting IgG1 with E430G mutation leads to the formation of hetero-hexamers that are more potent in mediating CDC than the corresponding homohexamers on their own or in combination (Figure 1B). This finding is exciting as it suggests that two $\mathrm{mAb}$ that target two different cell surface antigens may form bispecific hetero-hexamers in the membrane, effectively leading to target clustering and an increase in the density of C1q docking sites. It also sheds a light on a possible concerted MOA of polyclonal antibodies which might form hetero-hexamers if they target different cell surface antigens or different epitopes of the same cell surface antigen. Collectively, the study makes a strong case for investigating multispecific and multiparatopic biclonal, oligoclonal, and polyclonal antibodies for enhancing CDC compared to their parental $\mathrm{mAb}$. Finding co-operative target combinations, such as CD20 and CD37 in the current study, that enable hetero-hexamer formation in the presence or absence of hexamerization-enhancing mutations is a key challenge in applying this concept to other hematologic malignancies and solid tumors. In this context, hexameric monoclonal and hetero-hexameric biclonal antibodies should also be tested for enhancing other effector functions in addition to CDC. While research into bispecific antibodies has accelerated, with a huge increase in the number of related clinical trials that are now ongoing, ${ }^{15}$ polyclonal antibodies ${ }^{16}$ may well be the next wave of antibody-based cancer therapy. Hetero-hexamerization in the membrane is a possible MOA of polyclonal antibodies in IgG1 format, providing an incentive to investigate their therapeutic utility with and without hexamerization-inducing mutations.

In summary, Oostindie et al. ${ }^{1}$ make a compelling case for further exploration of hexamer-forming antibodies and the combination of two, or possibly even more, targeting $\mathrm{mAb}$. The advantages of antibody combinations might include not only increased cytotoxic activity, as described here, but possibly also better tumor-specific targeting and mitigation of tumor escape through antigen loss or target internalization. ${ }^{17}$ However, several aspects of this promising technology need further exploration. How effective is hexamer formation in vivo and what kind of hetero-hexamers might be formed, especially in tissue sites? The current study is limited to in vitro studies with CLL cells in suspension. It is not immediately clear how these observations will translate to in vivo settings. Furthermore, hetero-hexamers may form in different ratios, some containing equal ratios of antibodies, while in others one antibody may dominate. Will there be an optimal ratio and if so, could a desired composition be engineered into the antibody backbone? Translation of this promising approach into clinical trials may well constitute the next breakthrough in antibody therapy of B-cell malignancies. A first clinical trial with $\mathrm{mAb}$ engineered to facilitate hexamerization is ongoing in solid tumors (clinicaltrials.gov identifier: NCT03576131). GEN1029 (also called HexaBodyDR5/DR5) consists of a mixture of two mAb that bind to different epitopes on DR5 and activate this death receptor to induce apoptosis. Results from this and other studies of hexamerization-enhanced $\mathrm{mAb}$ and $\mathrm{mAb}$ combinations are eagerly awaited. 


\section{References}

1. Oostindie SC, van der Horst HJ, Lindorfer MA, et al. CD20 and CD37 antibodies synergize to activate complement by Fc-mediated clustering. Haematologica. 2019;104(9):1841-1852.

2. Diebolder CA, Beurskens FJ, de Jong RN, et al. Complement is activated by $\operatorname{IgG}$ hexamers assembled at the cell surface. Science. 2014;343(6176):1260-1263.

3. Salles G, Barrett M, Foa R, et al. Rituximab in B-cell hematologic malignancies: a review of 20 years of clinical experience. Adv Ther. 2017;34(10):2232-2273.

4. Zent CS, Victoria Wang X, Ketterling RP, et al. A phase II randomized trial comparing standard and low dose rituximab combined with alemtuzumab as initial treatment of progressive chronic lymphocytic leukemia in older patients: a trial of the ECOG-ACRIN cancer research group (E1908). Am J Hematol. 2016;91(3):308-312.

5. Witkowska M, Smolewski P, Robak T. Investigational therapies targeting CD37 for the treatment of B-cell lymphoid malignancies. Expert Opin Investig Drugs. 2018;27(2):171-177.

6. Yeung L, Hickey MJ, Wright MD. The many and varied roles of tetraspanins in immune cell recruitment and migration. Front Immunol. 2018;9:1644.

7. van Spriel AB, de Keijzer S, van der Schaaf A, et al. The tetraspanin CD37 orchestrates the alpha(4)beta(1) integrin-Akt signaling axis and supports long-lived plasma cell survival. Sci Signal. 2012:5(250):ra82.

8. Byrd JC, Pagel JM, Awan FT, et al. A phase 1 study evaluating the safety and tolerability of otlertuzumab, an anti-CD37 mono-specific ADAPTIR therapeutic protein in chronic lymphocytic leukemia. Blood. 2014;123(9):1302-1308.
9. Robak T, Hellmann A, Kloczko J, et al. Randomized phase 2 study of otlertuzumab and bendamustine versus bendamustine in patients with relapsed chronic lymphocytic leukaemia. Br J Haematol 2017;176(4):618-628

10. Stilgenbauer S, Aurran Schleinitz T, Eichhorst B, et al. Phase 1 firstin-human trial of the anti-CD37 antibody BI 836826 in relapsed/refractory chronic lymphocytic leukemia. Leukemia. 2019 May 14. [Epub ahead of print]

11. Weiner LM, Surana R, Wang S. Monoclonal antibodies: versatile platforms for cancer immunotherapy. Nat Rev Immunol. 2010;10(5):317-327.

12. Saxena A, Wu D. Advances in therapeutic Fc engineering - modulation of IgG-associated effector functions and serum half-life. Front Immunol. 2016;7:580.

13. de Jong RN, Beurskens FJ, Verploegen S, et al. A novel platform for the potentiation of therapeutic antibodies based on antigen-dependent formation of $\operatorname{IgG}$ hexamers at the cell surface. PLoS Biol. 2016;14(1):e1002344.

14. Cook EM, Lindorfer MA, van der Horst $\mathrm{H}$, et al. Antibodies that efficiently form hexamers upon antigen binding can induce complement-dependent ctotoxicity under complement-limiting conditions. J Immunol. 2016;197(5):1762-1775

15. Labrijn AF, Janmaat ML, Reichert JM, Parren P. Bispecific antibodies: a mechanistic review of the pipeline. Nat Rev Drug Discov. 2019 Jun 7. [Epub ahead of print]

16. Haurum JS. Recombinant polyclonal antibodies: the next generation of antibody therapeutics? Drug Discov Today. 2006;11(13-14):655660.

17. Taylor RP, Lindorfer MA. Fcgamma-receptor-mediated trogocytosis impacts $\mathrm{mAb}$-based therapies: historical precedence and recent developments. Blood. 2015;125(5):762-766.

\title{
The secret afterlife of platelets
}

\begin{abstract}
Nicholas A. Arce ${ }^{1,2}$ and Renhao $\mathrm{Li}^{1}$
'Aflac Cancer and Blood Disorders Center, Children's Healthcare of Atlanta, Department of Pediatrics, Emory University School of Medicine and 'Graduate Program of Molecular and Systems Pharmacology, Graduate Division of Biological and Biomedical Sciences, Emory University, Atlanta, GA, USA
\end{abstract}

E-mail: RENHAO LI - renhao.li@emory.edu

doi:10.3324/haematol.2019.224170

$\mathrm{P}$ atelets express a wide variety of receptors and signaling molecules that enable responses to diverse physiological and pathological stimulants. For instance, in normal hemostasis, exposure of subendothelial collagen may elicit platelet activation at the site of injury via glycoprotein (GP)VI, integrin $\alpha_{2} \beta_{1}$, and, through plasma von Willebrand factor, the GPIb-IX-V complex. Moreover, GPIb-IX-V in tandem with protease-activated receptors mediate thrombin-induced platelet signaling and activation. GPIba serves as a receptor for low concentrations of thrombin, transmitting a mechanosensory signal to mediate calcium-dependent 14-3-3 signaling while GPIb-IXdependent Rac1/LIMK1 signaling is modulated by protease-activated receptors. ${ }^{1,2}$ Upon activation, platelets aggregate and form clots that are interwoven with fibrin strands. Over the last several decades, much of the research effort has been focused on how platelets are rapidly activated by various agonists via their respective receptors and how activating, and sometimes inhibitory, signals amplify and propagate in the platelet. In most of these studies, the investigation ends at the cessation of blood flow, the formation of the clot, and/or the appearance of molecular signs that are well associated with platelet activation. A few minutes following platelet activation and aggregation, the blood clot contracts. In studies of clot contraction, the investigation often ends at the shrinkage of the platelet clot. ${ }^{3}$ However, little is known about the platelets in the clot following the contraction of the platelet/fibrin clot. In other words, after the formation of a stable blood clot, where do platelets go?

A study by Kim et al., published in this issue of Haematologica, demonstrates that after activation and contraction, thrombin-stimulated platelets break up into membrane particles, in a process termed platelet fragmentation. ${ }^{4}$ Thrombin is a major nexus between coagulation and platelet activation, as it generates fibrin to form a crosslinked fibrin plug and concurrently activates aforementioned receptors on the platelet surface. ${ }^{5}$ Platelet vesiculation and/or microparticle formation has been previously observed in response to thrombin and thrombin receptor activating peptide. ${ }^{6-8}$ The role that these platelet fragments play in hemostasis or platelet clearance has yet to be elucidated. In this new study, interestingly, Kim et al. observed a bimodal distribution of platelet fragments, the size of which can be attributed to the origin of the fragment. Filopodia as well as the main platelet body are two sources of platelet fragmentation, as smaller fragments were generated by filopodia, and larger fragments were generated from the cell body. Thus, it appears that platelet breakdown in response to thrombin stimulation is a regulated process of drastic morphological changes, platelet fragmentation, loss of function, and metabolic exhaustion. Platelet fragmentation may be a relatively newly discovered platelet behavior, adding to the ever-growing list of what 